\title{
TYPEN und FORSCHUNGSMETHODEN von VORURTEILEN (Eine Zusammenfassung)
}

Coşun SAN

\section{1- Vorurteil als soziales Phänomen:}

Die Individuen, Gruppen und Institutionen benరtigen Informationen aus der sozialen Umwelt, um ihre Verhaltensweisen den objektiven Gegebenheiten einer Situation anpassen zu können.

Wahmehmung, insbesondere aber die Informationsspeicherung und Handlunsplanung auf je cinzelne Umweltreize hin ist aus Gründen der Vielzahl der Einflüsse nicht moglich.

"Das menshliche Auge ist in der Lage ca. siebenmillionenfünfhunderttausend Farbwerte zu unterscheiden, während es in der englischen Sprache nur etwa vierhundert Farbwörter gibt. Und die meisten englischsprechenden Personen verwenden nur etwa zwölf dieser Wörter regelmăssig".

Dieses Beispiel zeigt deutlich, dass die Vielfalt der durch das Wahrnehmungsvermógen der. Menschen registrierten Umweltinformationen gezwungenerweise stark reduziert werden muss.

Dies geschieht durch Zusammenfassung der Umweltereignisse zu Klassen; d.h.: die Umwelt wird nach Kategorien, also Personen, Objekten und Ereignissen (oder deren ausgewăhlten typischen Altributen) geordnet. Diese Systematisierung setzt jedoch notwendigerweise Vereinfachungen (Lippmann, 1961, s. 88 ff., s. 148) voraus. Die ungeheure Menge an Informationen, die ein Individuum von seiner Umwelt und von den Auswirkungen seiner Handlungen empfangh, kann nur auf diese Weise zu brauchbaren Kategorien verabeitet werden. Sie erfüllen diese Funktion dadurch, dass sie die Umwelt a) sparsam und b) handlungsrelevant abbilden.

Die Kategorien als eine Art Schubladensystem werden von allen Individuen, Gruppen oder Institutionen nicht in gleicher Weise vervendet. Hinzu kommt, das für gleiche oder unterschiedliche Sachverhatte verschiedene, aber auch gleiche

*ord. Professor an der Fakultäı für Politische Wissenschaft der Universität Ankara 
Kategoriensysteme herangezogen werden. Kategoriensysteme und ihre Verwendung werden im Prozess der Sozialitisation gelernt.

Dieses soziale Phänomen, die Notwendigkeit des Kategorisierens, kann als grundlegender Prozess der Vonureilsbildung angesehen werden.

An dieser Stelle muss m. E. auch der Begriff "Idealtypus" kurz berührt werden. Die durch Kategorisierung (im oben genannten Sinne) gewonnenen Klassen stellen eindeutig Durchshnittstypen oder Häufigkeitstypen dar. Die sogenannten Idealtypen oder die reinen Typen im Weber'schen Sinne unterscheiden sich jedoch stark von den durch Kategorisierung erlangten Durchschnittstypen. In Max Webers eigenen Worten kommt ein Idealtypus "durch Steigenung bestimmter Elemente der Wirklichkeit" zustande, "durch Steigerung eines oder einiger Gesichtspunkte und durch Zusammenschluss einer Fülle von diffus und diskret, hier mehr, dort weniger, stellenweise gar nicht, vorhandener Einzelerscheinungen, die sich jenen einseitig herausgehobenen Gesichtspunkten fügen, zu einem in sich einheitlichem Gedankengebilde" (Weber, Wissenschaftslehre, S. 146 ff., $190 \mathrm{ff}$.). Inhaltlich trăgt diese Konstruktion den Charakter einer Utopie in sich, die durch gedankliche Steigerung bestimmter Elemente der Wirklichkeit entnommen sind. Der Idealtypus ist dennoch kein Vorbild, sondern durch Auswählen und Weglassen von unwichtig erachteten Einzelmerkmalen theoretisch gewonnenes Gedankenbild, dem die konkereten Gebilde, Prozesse, Handlungen usw. nach ihren typischen Charakteristika zugeodnet werden kőnnen. Auf diese Weise dienen die Idealtypen dazu, die Abweichungen der in der Realită vorhandenen sozialen Situationen von diese festzustellen. Der Idealtypus ist also cin Messgerät, eine Methode.

Was für unsere Betrachtungsweise in diesem Zusammenhang von Bedeutung ish, ist die Aehnlichkeit der Prozesse der "Idealtypenbildung" und "Vorurteilsbildung". Dies heisst wiederum nicht, dass Idealtypen und Vorurteile ausser der Aehnlichkeit der Bildungsprozesse irgendwelche Parallelitäten aufweisen.

Zusammengefasst kann behauptet werden, das Vorurteile Nebenprodukte des sonst für das Individuum sehr nützlichen und unentbehrlichen Kategorisierens sind und hinsichtlich der Bildungsprozesse mit Idealtypen gewisse methodische Achnlichkeiten zeigen.

\section{2- Der Begriff "Vorurteil":}

Die Verwendung des Oberbegriffs "Urteil" geht offenkundig von der in vielen Sprachen ăquivalenten Vokabel Vor-Urteil aus: (lat:: praeiudicium; eng.: prejudice/prejudgement; frz.: préjugé etc) (Ginsberg, 1964, s. 167 ff.).

Im allgemeinen werden die meist abstrakten Begriffsinhalte im sozialwissenschaftlichen Bereich nicht uniform definiert und zwar aus zwei wichtigen Grüinden:

a- Die letzten 150 Jahre unserer Geschichte sind durch einen raschen gesellschaftlichen und kulturellen Wandel gekennzeichnet. Dieser Wandel betrifft die Produktionsweise, den Lebensstandard, die Herrschaftsverhäl tnisse ebenso aber moralische Normen und allgemeine Grundüberzeugungen. 
Diese Tatsache führt dazu, dass die damit zusammenhăngenden Begriffe die soziale Realităt nur zum Teil reflektieren können. Sogar kommt es manchmal vor, dass sie einfach zu leeren Phrasen werden und ihre Funktion als Begriff vollig verlieren.

b- Andererseits ist es durchaus wissenschaftlich und legitim, wenn Wissenschafter nur bestimmte Aspekte einer sozialen Situation hervorheben und diese zu analysieren oder zu erforschen versuchen. In vielen Fällen scheint es sogar notwendig zu sein, den eigenen Begriffsapparat durch Heranziehung von geeigneten wissenschaftlichen Argumenten neu zu bilden.

Von diesem Standpunkt aus, dürfte es keine Überraschung sein, im sozialwissenschaftlichen Bereich gleiche Begriffe mit voneinander abweichenden Definitionen zu treffen.

Die eben erwähnte Tendenz ist auch hinsichtlich des Begriffes "Vorurteil" festzustellen, so dass die Vielfalt der Vorurteilsdefinitionen nur unter Kategorienklassen . zu erfassen sind:

In der soziologischen und sozialpsychologischen Literatur überwiegt bei den angebotenen Definitionen der Terminus "Einstellung" (attitude) als Oberbegriff (Ginsberg, 1964, s. 167). Vorurteile werden aber auch in anderen sozial wissenchaftlichen Bezugssystemen analysiert: statt des Einstellungskonzepts als einem Bezugssystem zur Erklärung individuellen sozialen Verhaltens werden vor allem solche zur Erklärung der "Beziehungen zwischen Gruppen" herangezogen.

Ohne auf eine tiefgreifende Analyse der Vorurteilsdefinitionen einzugehen, könnte man für die Einstellugsdefinitionen ein typisches Beispiel nennen: "Das Vorurteil ist eine Form von Feindscligkeiten in zwischenmenschlichen Beziehungen" (Ackermann u. Jahoda, 1950, s. 3 ff.)

Dic Gruppenbezogenheit des Vorurteilsbegriffs kommt z. B. in dieser Definition zum Ausdruck: "Das Vorurteil... bezieht sich auf das Verhälınis von Mitgliedern einer bestimmten Binnengruppe (in-group) zu einer oder mehreren von den dieser Gruppe entsprechenden Fremdgruppe (out-group). Unter in-group versteht man cine Gruppe, zu welcher der einzelne in einer so nahen Beziehung steht, dass er sie als "wir" oder "uns" bezeichnet, wogegen er sich auf dic out-group.... als "sie" bezicht" (Heintz, 1957, S. 14, ff.).

Eine १ctzte Definition (historisch gesehen aber einer der ersten) des Vorurteils im weitesten Sinne des Wortes wäre: "Vorurteile sind jene Urteile, die man ohne genügende Kenntnis und Überprüfung eines Sachverhalts fällt oder kritiklos weiterverbreitet" (Jaucourt, 1765, in: Encyclopédie).

\section{3- Arten sozialer Vorurteile:}

\section{a- Merkmale und Funktion von Vorurteilen:}

Die Merkmale der Vorurteile sind am leichtesten im Falle der rassischen oder ethnischen Vorurteile zu sehen: 
aa-Zunächst ist zu nennen die unkritische Verallgemeinerung, die dazu führt, allen Angehơrigen einer Gruppe Eigenschaften zuzuschreiben, die eigentlich nur bei einigen wenigen zu finden sind (Faulheit).

bb- Dann sind zu nennen das auswăhlende Hervorheben, die Tendenz also, bestimmte Eigenshaften áls besondere Charakteristika einer Gruppe anzusehen, die in Wahrheit ebenso allgemein auch in anderen Gruppen zu finden sind (Reizbarkeit).

cc- Es folgt das Weglassen, die Tendenz also, bei Gruppen, denen mit Ablehnung begegnet wird, erstrebenswerte Eigenschaften entweder zu übersehen oder aber, wenn diese zu offensichlich sind, um verleugnet zu werden, sie als untypisch beiseite zu schieben (... zwar sind manchmal manche relativ fleissig, aber...),

dd- Schliesslich die Diskriminierung, d.h., die Tendenz, bei einer Gruppe Handlungen zu verdammen, die bei anderen vergeben oder übersehen gar gerühmt werden (Betrug-ein ideenreicher Betrug).

ee- Endlich sind einige weitere Faktoren wie Beeinflussbarkeit, bewusste oder unbewusste Selbstuăuschung und die Neigung zu überspitzten und logisch-vereinfachten Aeusserungen von Bedeutung (Ginsberg, 1963, S. 16 ff.).

Die sozialen und nationalen Vorurteile haben, wie mehrmals erwăhnt, auch eine Reihe wichtiger Funktionen zu erfüllen.

An erster Stelle ist hier das Vorurteil als Instrument der sozialen Orientierung zu nennen. "Ein Volk ohne Vorurteile, ein Volk mit vollig neutraler Haltung, wăre in jeder erdenklichen Kultur unvorstellbar," (Lippmann, 1964, S. 88), da die sozialen und nationalen Vorurteile, positiv gesehen, den Zusammenhalt einer Gesellschaft oder einer Gruppe stărken (Legenden, Mărchen u. ă). Angehörige einer bestimmten Gruppe entwerfen von sich und ihrem sozialen Gebilde ein vorurteilvolles überhöhtes Selbstbild (Autostreotyp).

Unter negativem Aspekt führen Vorurteile zu einem Fehlverstăndnis der sozialen Wirklichkeit. Meinungsstreotypen und Klischeevorstellungen des vulgärsoziologisçen Horizonts begünstigen nicht nur Sclbsttăuschung, sondern auch die Aufstellung von Fremdbildern (Heterostreotypen) einer fremden Gruppe, wonach deren Angehörige als falsch, hinterlistig, minderwertig, ausbeuterisch usw. gelten. Hellenen-Barbaren, Kulturvőlker-Naturvolker, Industricländer-Entwicklungsländer etc. wăren die globalen Unterscheidungen, die die Qualităten des Eigenbildes und die Geringschătzung des Fremdbildes widerspiegeln.

\section{b. Entstehung und Haltbarkeit von Vorurteilen:}

aa- Die Kultur: Die Normen, Traditionen und anderen kulturellen Überliefcrungen der Gesellschaft stellen wohl einer der wesentlichsten Faktoren dar, die dafür verantwortlich sind, dass Vorurteile übernommen, weiterhin aufrechterhalten und weitergeleitet werden. Hier sind von allem zu nennen Literatur und Theater sowie die modemen Massenmedien wie Presse, Rundfunk, Film und Femsehen. 
bb- Die Familie: Sie ist von besonderer Wichtigkeit bei der-Bildung sozialer Einstellungen der Kinder, wobei soziale Schichtzugehörigkeit und formales Bildungsniveau der älteren Familienmitglieder eine entscheidende Rolle spielen.

cc- Die Schule: Hier muss besonders auf die Bedeutung der Lehrbücher über Geographie, Geschichte und Sprachen verwiesen werden. Sie vor allem vermitteln einen .ersten Eindruck von anderen Volkem. Dieses Bild ist nicht immer differenziert und objektiv; häufig enthalt es Elemente der Streotypie oder gar abwertende Vorstellungen von anderen Gruppen. Das Wissen, das geboten wird, ist meist die bestehende, herrschende Meinung der Gesellschaft, einschliesslich ihrer Fehleinstellungen und Intümer.

dd- Der Staat: Nicht nur in den autoritaren sondern auch in den sogenannten freiheitlich demokratischen Regimen werden oft Vorurteile in Form von Feindbildern als .Instrument bestimmter politischen Zielsetzungen erzeugt, die sogar nach der Erreichung dieser Ziele in den breiten Massen einer jeden Gesellschaft weiterleben, obwohl die meist künstlich konstruierte Kausalităt nicht mehr besteht.

Auserdem ziehen wir im alltäglichen Leben ständig Kategorien und Vorurteile heran, um unsere soziale Umwelt besser zu erkennen und uns danach zu richten. Schliessen wir neue Bekanntschaften oder begegnen wir einem Mann oder einer Frau zum ersten Mal, dann häufen sich kategoriehafte Vorurteile an und wir versuchen, anhand dieser Referenzschubladen diese besser kennenzulemen (Physiognomie: schlank-fett, kleingross, alt-jung, weiblich-männlich, laut-leise, kaltblütig-temparamentvoll etc.).

Die Phänomenologie des Vorurteils im allgemeinen sagt uns, dass Vorurteile zum Haltbarsten in der menschlichen Geschichte gehören. In Legenden, Märchen, Erzăhlungen, Sprichwörtern und Anektoden leben manchmal Vorurteile weiter, obwohl die daraufgezielten sozialen Gruppen oder ethnischen Minderheiten schon längst nicht mehr existieren. Nur bedeutsame Veränderungen wirtschafulicher, politischer und sozialer Art zwischen Gruppen und Nationen könnten zu einer Modifikation der bisherigen Vorurteile führen.

Auch traumatische persơnliche Erfahrungen und intensive Umerziehung im ăusseren Rahmen einer radikalen Demokratisierung der Gesamtgesellschaft könnten eine Menge dazu beitragen.

\section{c- Klassifizierung von Vorurteilen:}

Ausser der Zweitteilung von Vorurteilen in negative und positive scheint eine ausschöpfende Klassifizierung von Vorurteilen nicht moglich zu sein.

Die oft festzustellenden Vorurteile sind: werden:

aa- Nationale oder ethnische Vorurteile, welche auch als Stereotype bezeichnet

bb- Vorurteile, die auf geschlechulicher Diskriminierung beruhen (Mann-Frau);

cc-Vorurteile, die sich auf Generationskonflikte stützen (alt-jung); 
dd- Vorurteile, die sich auf soziale Differenzen beziehen (soziale Schichtszugehơrigkeit, Berufsunterschiede usw.).

All diese Vorurteile treten in der Regel im Zusammenhang mit Differenzen und Konflikten zwischen verschiedenen sozialen Kategorien, Gruppen und Schichten auf, die sich aus dem Bestand sozialer Privilegien und sozialer Benachteiligungen ergeben. Die Unterschiede zwischen Kategorien, Vorurteile und Streotypen könnten anhand des unten stehenden Beispiels veranschaulicht werden:

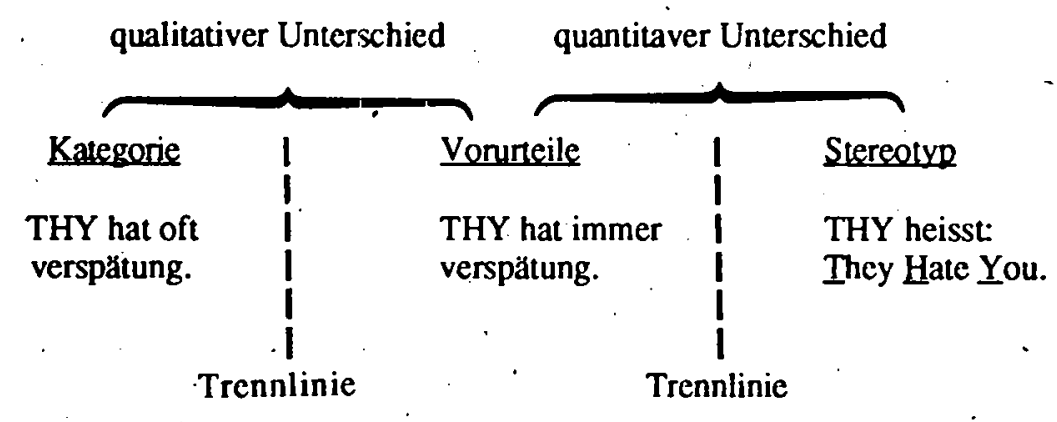

\section{4- Methoden zur Vorurteilsforschung:}

\section{a- Im allgemeinen:}

Methode bedeutet im weitesten Sinne Art und Weise, Wege und Mittel, derer sich der Forscher bedient, um zu Ergebnissen von wissenschaftlicher Zuverlässigkeit zu gelangen.

Alle Wissenschaftszweige, Naturwissenschaft sowie Sozialwissenschaft, brauchen die theoretische Grundlegung und Durchdringung. Um wissenschaftlicher Gültigkeit willen müssen theoretische Arısătze und Überlegungen durch gegenstandsgerechte empirische Untersuchungen überprüfbar sein, sich also als richtig oder falsch erweisen kőnnen. Umgekehrt werden die Theorienbildung, das wissenschafuliche Verständnis und die Sinnerschliessung menschlichen Lebens erst durch die empirische Erfassung der konkreten gesellschaftlichen Verhältnisse ermöglicht (Wallner, 1972, S. 44).

\section{b- Verschiedene Ebenen der Sozialforschung:}

\section{aa- Betrachtungsweisen:}

Betrachtungsweise heisst die Entscheidung, nach welchen Verfahrensgrundsătzen und mil Hilfe welcher angemessener Untersuchungstechniken ein ausgewähltes soziales Problem erforscht werden soll.

- Empirische Untersuchung, z. B. mit Hilfe von Intervicws/Fragebogen.

- Herausarbeitung eines Idealtypus.

- Dialektische Betrachtungsweise. 
- Werffreiheit.

(Deduktiv-induktiver Schluss kann nur in der Logik angewandt werden)

bb- Verfahrensgrundsätze:

Die Verfahrensgrundsătze werden hăufig mit Methoden im engsen Sinne gleichgesetzt. Gegenwărtig herrscht Unklarheit über die Begriffśdeutung von Methode und Technik (Generell-speziell).

Einige Beispiele hierzu:

- theoretisch - empirisch

- verstehende - kausalerklärende

- analytische - synthetische

- statistische - deskriptive

Methoden im engeren Sinn

\section{cc- Forschungstechniken:}

Die Techniken umfassen im wesentlichen die Mittel und Massnahmen, die bei der Durchführung soziologischer Untersuchungen', insbesondere nach empirischen, kausalerklărenden, analytischen, statistischen und deskriptiven Verfahrensgrundsătzen angewandt werden.

Die am meisten angewandten Techniken sind:

Beobachtung, Befrafung-Interview, Gruppendiskussion, Experiment, Soziometrie, Skalierung, Erhebungsauswahl-Stichprobe-Sample, Panel-und Trenderhebungen, Einzelfallsudie, Inhaltsanalyse und Faktorenanalyse.

Für Vorurteilsforschung kămen fast all diese Techniken in Frage. Bevorzugt sind aber in erster Linie die Beobachtung, die Befragung und die Inhaltsanalyse.

Eine emprirische Untersuchung eines bestimmten sozialen Sachverhalts erfolgt in der Regel in aufeinander Folgenden 4 Phasen:

(1) Wahl eines Themas und seine theoretische Umreissung.

(2) Entwurf der Hypothesen und Wahl der geeigneten.

Methoden und Techniken.

(3) Anwendung der Techniken.

(4) Interpretation der Materialien.

b- Methoden und Techniken zur Vorurteilsforschung:

aa- Die -Beobachtung:

Beobachtung ist die bewusst geplante und protokollarisch niedergelegte Erfassung von wahrnehmbaren Sachverhalten der sozialen Welt. Durch Planung wird die 
Beobachtung aus der Allagserfahrung auf eine wissenschaftliche Ebene gehoben und zugleich eine Kontrolle ermöglicht.

Die Zuverlässigkeit und Genauigkeit der durch Beobachtung gewonnenen Ergebnisse hängen u.a. ab:

(1) Von der Beobachtbarkeit eines Tatbestandes.

(2) Von der Beobachtungssituation (Schule, Arbeitsplatz)

(3) Vom Standort des Beobachters etc.

Diese Technik findet vorallem beim Experiment (Laboratoriumexperiment und Feldexperiment volle Anwendung (Atteslander, 1975, S. 136 ff.).

\section{bb- Die Befragung-das Inteview:}

Befragung ist die bewusst geplante und in Protokollen festgehaltene Erfassung von sozialen Sachverhalten, Meinungen, Urteilen, Beweggründen des Handelns usw. mit Hiilfe mündlicher oder schriftlicher Beantwortung von Fragen, die einem bestimmten Personenkreis gestellt werden.

Die Befragung ist gegenwärtig eine der am weistesten verbreiteten Techniken zur Gewinnung von Informationen über die soziale Welt.

Die Stichhaltigkeit der Angaben hăngt ab von der Persönlichkeit der Befrager, der gründlichen Vorbereitung, dem planmässigen Ablauf der Befragung und der Bereitwilligkeit der Befragten zu Auskünften. Ein besonderes Problem stellt die Ausschaltung der Beeinflussbarkeit von Ergebnissen durch die Befrager dar (Alteslander, 1975 , S. 85 ff.).

\section{cc- Die Inhaltsanalyse:}

Die Inhaltsanalyse hat sich als eine Forschungstechnik in den Sozialwissenschaften durchgesetzt. Sie entwickelte sich aus der alten qualitativen Interpretation, wie sie in den Geschichts-und Geisteswissenschaften üblich ist, entstand also hauptsächlich aus Erklärung und Auslegung von Texten. Auf verschiedenen Entwicklungsstufen wurde sic allmăhlich in den Bereich des Quantitativen erweitert.

Das Verfahren der Inhaltsanalyse wurde in erster Linie für die Zwecke der Untersuchung der Massenmedien ausgearbeitet; es ist jedoch auch anwendbar auf andere Erscheinungsformen allgemeiner Kommunikation, wie z.B. auf persönliche Dokumente und Briefe, Interviews und Gespräche usw. Unbestritten ist aber die Hauptanwendung der Inhaltsanalyse auf die vier klassischen Medien: Presse, Film, Rundfunk und Femsehen (Ritsert, 1972, S. 14 ff.)

1974 schlug H.D. Lasswell einen fünfteiligen Fragenkatalog zur Beschreibung des bis dahin kaum spezifisierten Kommunikationsprozesse vor. Diese fünf Fragen, die sogennte. "Lasswell-Formel", sind heute als theoretischer Rahmen des Kommunikationsprozesses allgemein anerkannt; sie werden hăufig zitiert oder in abgeänderter Form wiedergegeben: "Who says what in which channel to whom with what . effect?" (Wer sagt was auf welchem Weg wie zu wem mit welcher Wirkung?). 


$\begin{array}{lll}\text { wer } & \begin{array}{l}\text { control analysis } \\ \text { content analysis }\end{array} \text { Schwerpunkt } \\ \text { was } & \text { media analysis } & \\ \text { wie } & \text { audience analysis } & \\ \text { wem } & \\ \text { weicher Wirkung } & \text { effect analysis }\end{array}$

Es ist eine Selbsverständlichkeit, dass sich die Untersuchugstechniken der Inhaltsanalyse nicht auf eine Beschreibung von Aeuserungen menschlichen Verhaltens beschränken. Sie zielen vielmehr ab:

(1) auf die Erkundung der sozialen Motivationen und Charakteristika der Urheber dieser Aeuserungen;

(2) auf die Ermitulung der sozial-relevanten Reaktionen, zu denen diese Aeuserungen als Reize herausfordem.

Der Inhaltsanalyse können unterzogen werden: kulturelle und soziale Denksysteme aufgrund von philosophischen Publikationen, von religiösem Schriftum, von Kunstwerken etc.; Dichtung, Massenkommunikationsmittel, wie Presse, Film, Rundfunk und Fernsehen; Propagandamaterial; Meinungsstreotypen; Vorurteile usw.

Dabei von grősster Bedeutung sind inhaltlich: leitbildhafte Vorstellungen, bevorzugte Themenkreise, Wahl suggestivkräftiger Ausdrücke, Satzbau, Symbole etc. Die Inhaltsanalyse eröffnet Möglichkeiten für Rückschlüsse auf den Horizont und die Bewusstseinslage einer Gruppe oder einer Gesamtgesellschaft (Ritsert, 1972, S. 14 ff.). 